Article

\title{
Performance Assessment of a Developed Brake Frame Based on the Application of a Proposed Equivalent Model in a Net Protection System for Debris Flow
}

\author{
Sungtae Kim, Sanghyun Hong, Jungjoo Kim and Hankyu Yoo * \\ Department of Civil and Environmental Engineering, Hanyang University, 55 Hanyangdaehak-ro, Sangnok-gu, \\ Ansan 15588, Korea; kst@dhft.co.kr (S.K.); jerom1st@hanyang.ac.kr (S.H.); lineup011@hanyang.ac.kr (J.K.) \\ * Correspondence: hankyu@hanyang.ac.kr; Tel.: +82-31-400-5147; Fax: +82-31-409-4104
}

Received: 13 June 2018; Accepted: 5 July 2018; Published: 8 July 2018

\begin{abstract}
The demand for a net protection system for mitigating debris flow has increased recently for which new energy absorption devices have been hereby developed. The purpose of this study is to analyze the behavior and energy absorption rate of a brake frame, to find an efficient approach for its numerical simulation, and to increase its field applicability by analyzing its effects. Three methodologies were used for this purpose. Firstly, quasi-static load tests were conducted for analyzing the behavior of the brake frame. Secondly, three-dimensional FE modeling was performed to analyze the buckling and fractural behavior of steel rings. Lastly, the effects of the brake frame were numerically analyzed based on a proposed equivalent model. The results show that the brake frame can withstand the external force by elastic/local buckling, fracture of a steel ring, and elastic/plastic behavior of wire rope, and has the energy absorption rate of $53 \mathrm{~kJ}$. It is deduced that the proposed equivalent model is capable to accurately simulate the behavior of the brake frame and can replace the three-dimensional model. The tensile force reduction in the system by using brake frames was observed to be about $75 \%$. It is concluded that the proposed equivalent model for brake frame design is practically applicable.
\end{abstract}

Keywords: debris flow; net protection system; brake frame; quasi-static load test; buckling; equivalent model; three-dimensional FE model; anchor capacity; impact energy

\section{Introduction}

The sudden phenomenon of debris flow is quite destructive and occurs due to climate change. It causes damage to the people, property and structures [1]. In order to minimize the damage, research work is currently going on to predict the phenomenon of debris flow and develop its countermeasures, such as protection systems. The protection system for the debris flow can be classified into closed-type (such as concrete gravity dams) and open-type protection (such as buttress dams). The design standard of the system depends on the amount of debris flow and its pattern of the flow [2]. However, these two traditional protection systems have their own limitations, such as the need for a strong foundation for construction on steep slopes and it is also difficult for heavy machinery to access the construction site. Therefore, some net-type protection systems have been developed recently which depend on the flexibility of the net.

For this study, the developed net protection system mainly consists of a net, energy absorption devices namely brake frames, and anchors, as shown in Figure 1. All the elements of the net protection system were designed carefully according to the design details so that these element could be saved from possible damage due to debris flow impact. The net is a kind of mesh or web made by wire ropes and it is designed to contain the debris flow by blocking and holding the material within itself. 
The importance of energy absorption devices in the net protection system is to absorb the kinetic energy from the debris flow forcing it to make a halt. Recently, various energy dissipating devices have been introduced in Italy which are capable of absorbing $100 \mathrm{~kJ}$ to $5000 \mathrm{~kJ}$ impact energy [3]. The numerical approach is widely used to assess the performance and effects of these devices while developing the constitutive laws and models for each element of the system and subsequent comparison with the experimental results [3,4]. An analytical model for a flexible debris flow protection system was suggested due to the difficulty in the dynamic analysis and the consideration of non-linearity of the structures [5].

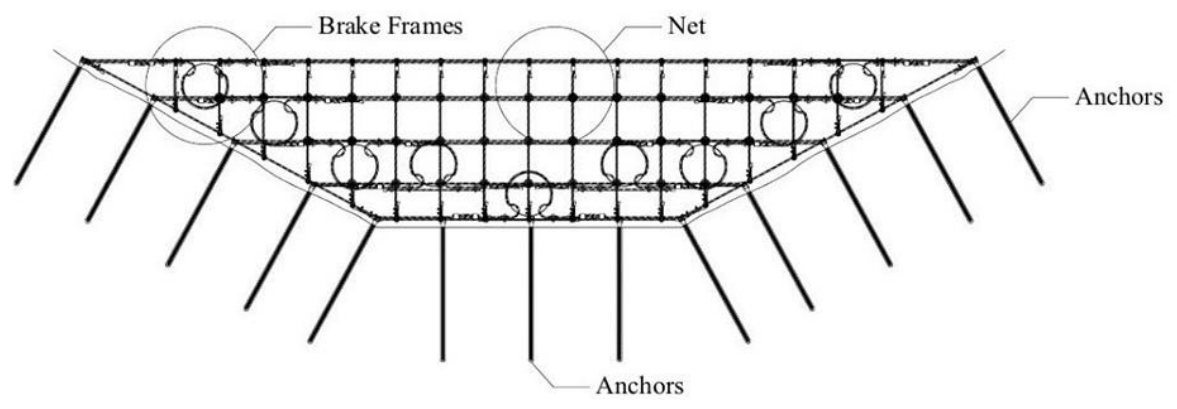

Figure 1. Transverse cross-section of the developed net protection system.

Anchor supports play an important role in the system by transferring the kinetic energy from the impact to the system, and the anchor capacity can be determined by observing the interaction between the impact energy of the debris flow and the energy absorption devices. The performance of the energy absorption devices has to be assessed before the field application because the anchor support capacity varies with the efficiency of the energy absorption devices. There are many studies related to the full-scale impact tests and physical model tests that analyze the behavior of net protection systems as per their anchor capacity [6-10]. The anchor design primarily depends on the slope condition, engineering judgment, and information from previous experiences [11]. The load transfer and failure characteristics of the anchors used in net protection system were investigated under vertical and horizontal loads based on field test [12]. The most important aspect is the analysis of the systematic relationship between each element.

The purpose of this study is to assess the performance of developed brake frames before field application and to ensure the stability of the protection system through predicting the impact load on the system. Therefore, an investigation scheme was developed, as explained below. Energy dissipation is the area under the force-displacement curve obtained during quasi-static load tests. Firstly, a brake frame was developed for providing up to $53 \mathrm{~kJ}$ of energy dissipation and then quasi-static load tests were performed to analyze the behavioral characteristics of the brake frame, and also to analyze its energy absorption capacity, as explained in Section 2. During the quasi-static load tests, permanent deformation was observed in one of the steel rings provided in the brake frame due to buckling and tensile fracture, resulting in energy dissipation in each brake frame. These tests proved to be more economical since a single brake frame used in this study was capable to deform up to $1.5 \mathrm{~m}$ which is comparatively greater than other devices.

Subsequently, the behavior and deformation of each of the system components was recorded and an equivalent model was, thus, proposed for the validation of numerical simulations in different conditions, as discussed in Section 3. The proposed equivalent model is based on the force-displacement relationship and it can be used to simulate the complex behaviors of the brake frame, including buckling and metal fracture, using a one-dimensional model. This model can be used to increase the efficiency of numerical modeling in this study.

Furthermore, in Section 4, the effects of using a brake frame in the system were analyzed through the numerical approach by comparing its results with those of a system without which the brake frame 
and the tensile force was observed to be significantly decreased in the wire rope due to the use of the brake frame.

\section{Development of a Brake Frame for Energy Absorption}

\subsection{Development of a Brake Frame}

This brake frame plays an important role in stopping the impact block as well as in reducing the stresses on the wire rope (net protection system) and thereby decreasing the tensile load on the anchor by absorbing the impact energy. This study includes the development of a brake frame that consists of the components such as wire rope, guide rope, steel ring, and U-clips, as shown in Figure 2. An independent wire rope core was used as the wire rope which was designed by using $16 \mathrm{~mm}$ diameter. The steel ring is a representative element of the net protection system in which large permanent deformation occurs. For this study, four holes of $20 \mathrm{~mm}$ diameter were drilled through the steel ring. The wire rope of $2900 \mathrm{~mm}$ length was attached to the periphery of the steel ring to create effective bonding and to share the tensile strength of wire rope with the steel ring. This was achieved by passing the wire rope in and out through the drilled holes and further connecting it to the steel ring by using four U-bolts. A $50 \mathrm{~mm}$ wide and $4 \mathrm{~mm}$ thick portion of SS400 (related to tensile strength of $400 \mathrm{MPa}$ ) steel was used to assemble the $300 \mathrm{~mm}$ diameter steel ring. The steel ring was designed to be fractured with an increase in the tensile strength and a fracture point was designated manually to the ring so that elongation of the wire rope occurs when the steel ring breaks apart, thereby enabling the brake frame system to absorb the impact energy. In order to assist the fracture initiation and propagation on the steel ring, a triangular groove with $15 \mathrm{~mm}$ breadth and $15 \mathrm{~mm}$ height was created on the periphery of steel ring. The wire rope was further attached to a guide rope of $1500 \mathrm{~mm}$ length using four or six U-clips.

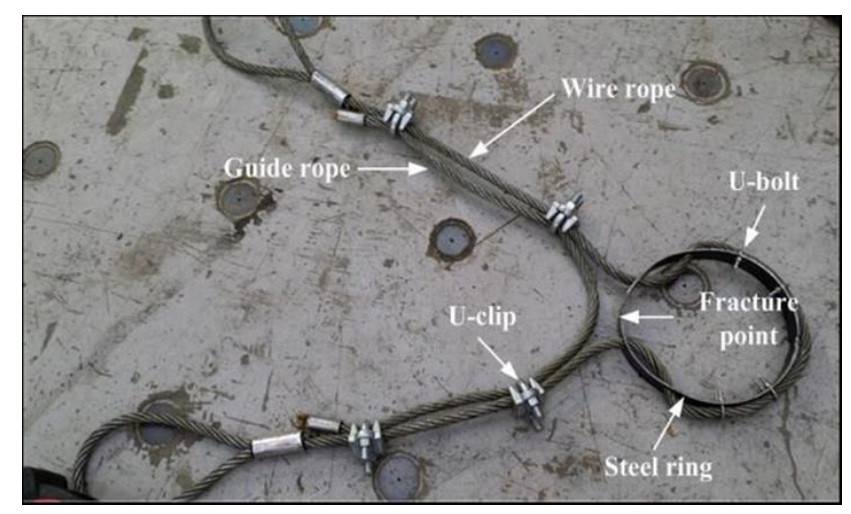

(a)

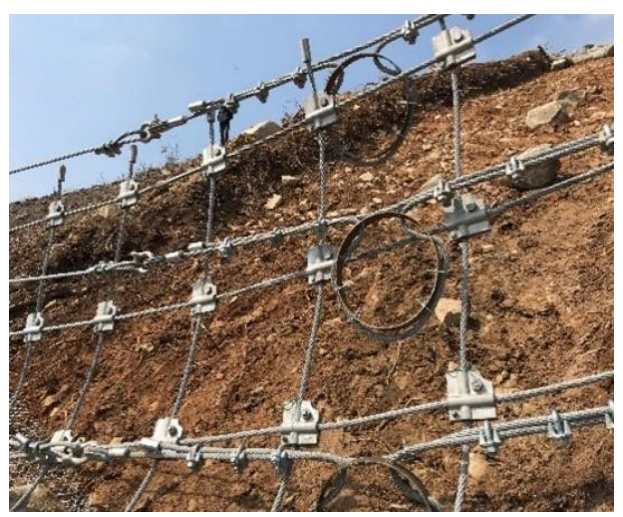

(b)

Figure 2. Depiction of the elements of brake frame and installation in the system, (a) components of brake frame; and (b) brake frame installed in the system.

\subsection{Structural Performance of the Developed Brake Frame}

In order to observe the structural performance, and also to analyze the absorbing rate of the brake frame, quasi-static load tests were performed in the hybrid structural testing center located at Myongji University, South Korea. A $5000 \mathrm{kN}$ static actuator was used to develop $3500 \mathrm{kN}$ tensile force in the brake frame and the piston speed was maintained as $250 \mathrm{~mm} / \mathrm{min}$. Subsequently, two types of tests were performed using two different sets of U-clips attached on the brake frame, as shown in Figure 3.

The relationship between tensile force and displacement developed in the brake frame is shown in Figure 4. The behavioral characteristics of the brake frame during the tests can be divided into six phases. The first phase consists of the resistance of U-clips in which the displacement was observed to be approximately $0-480 \mathrm{~mm}$. In this displacement range, the peak tensile force was dependent 
on the number of U-clips used and the tightening of bolts (workability) resulting in different results observed in test 1 and test 2 . The second and third phases are named as elastic/local buckling and plastic collapse of the steel ring, respectively. The displacement was observed to be approximately $480-700 \mathrm{~mm}$ in the phase of elastic/local buckling which was developed in both shoulders of the steel ring. Afterwards, plastic collapse occurred around the displacement range of 700-900 mm. Tensile failure of the steel ring was observed in phase four. Tensile failure occurred at $72-74 \mathrm{kN}$ at the fracture point of the steel ring regardless of the number of U-clips used. In the phase five, plastic behavior was observed in the brake frame in the displacement range of $1100-1400 \mathrm{~mm}$ in both tests. In the final phase, elastic and plastic behaviors were observed in the wire rope after the displacement crossed the $1400 \mathrm{~mm}$ limit. The tensile failure of the wire rope was developed around 176-192 kN. The behavior of the brake frame in all of the six phases is illustrated in Figure 5.

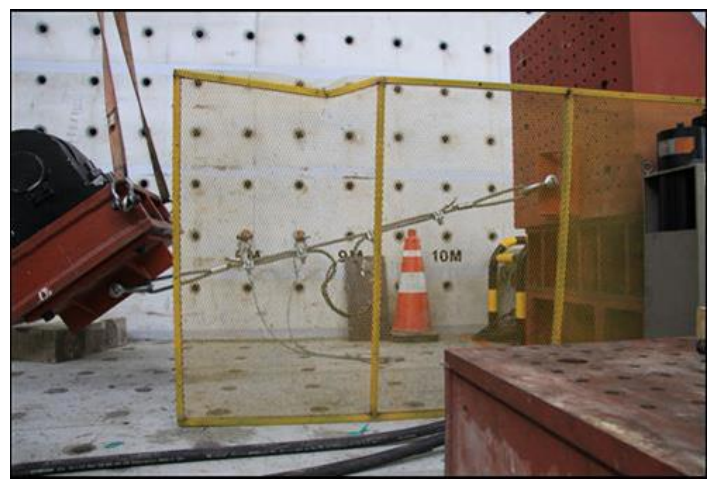

(a)

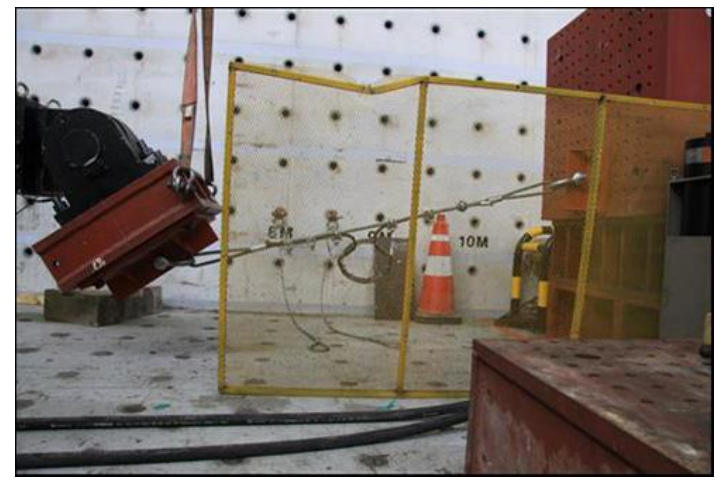

(b)

Figure 3. Quasi-static load tests using different sets of U-clips, (a) brake frame using four U-clips; and (b) brake frame using six U-clips.

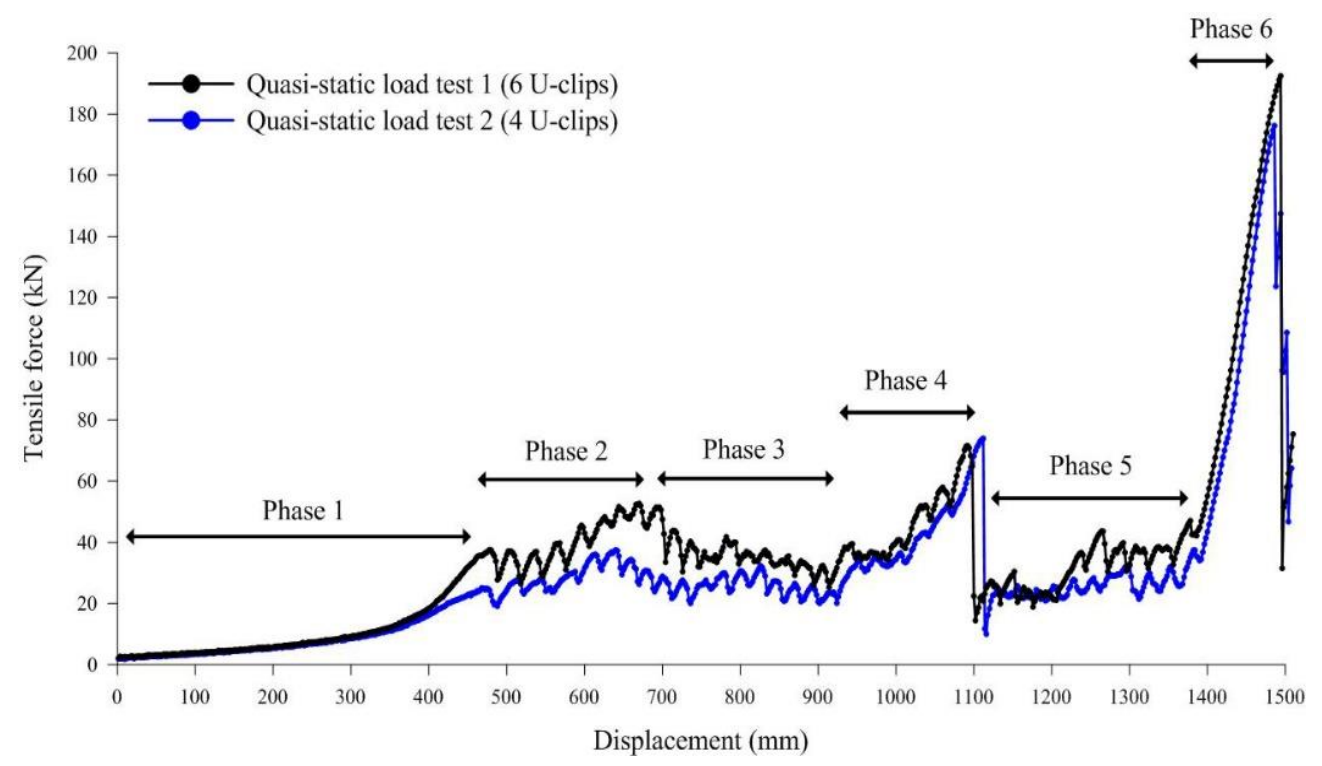

Figure 4. Classification of quasi-static load tests result in six phases. 


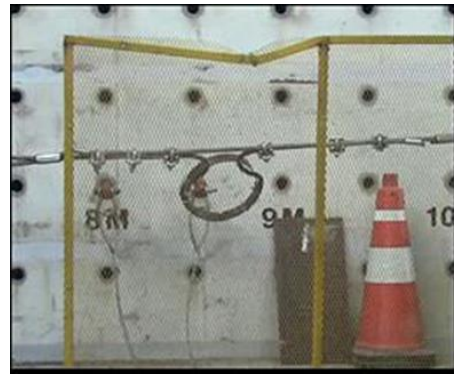

(a)

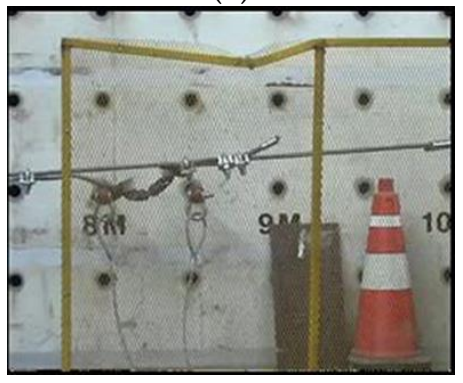

(d)

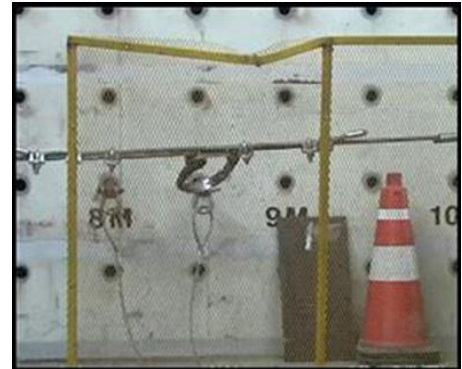

(b)

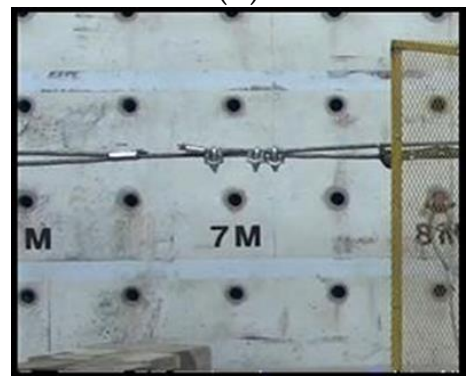

(e)

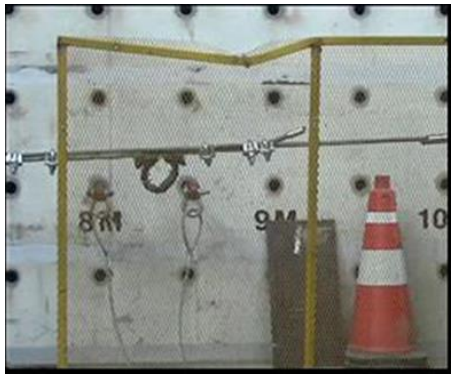

(c)

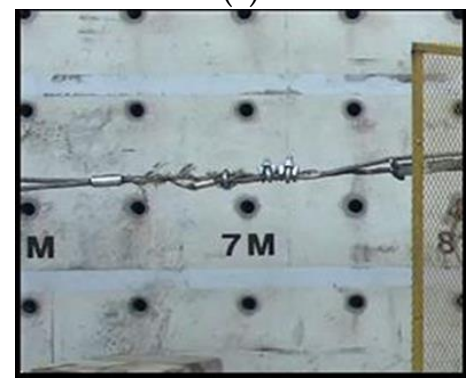

(f)

Figure 5. Illustration of deformation phases of the brake frame, (a) phase 1; (b) phase 2; (c) phase 3; (d) phase 4; (e) phase 5; and (f) phase 6.

\section{Equivalent Model for Brake Frame}

\subsection{Numerical Modeling for Three-Dimensional Behavior}

In this study, three-dimensional FE model was used to analyze the behavior of brake frame for proposing an equivalent model for its analysis and a comparison was made with the results of quasi-static load tests. As shown in Figure 6, the 3-D FE models include the steel ring being modelled as a shell element and wire rope being modelled as a truss element. The steel ring was modelled as a four-noded, doubly-curved, thin shell element (S4R), and was divided in to a total of 7312 elements which had $2.5 \mathrm{~mm}$ spacing between each other. The wire rope was modelled as a two-noded linear 3-D truss element (T3D2), and was divided in to a total of 716 elements which had $2 \mathrm{~mm}$ spacing between each other. The numerical analysis was performed with ABAQUS Explicit (SIMULIA, Johnston, RL, USA 2016) [13].

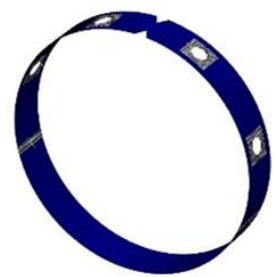

(a)

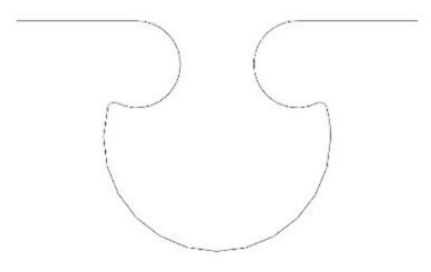

(b)

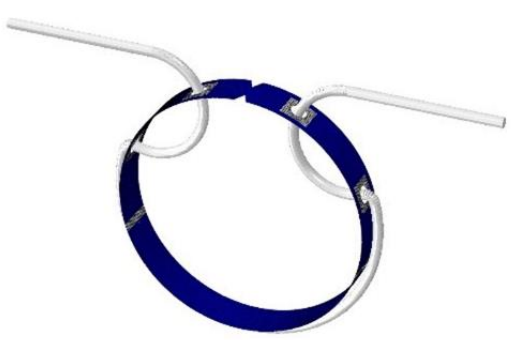

(c)

Figure 6. Three-dimensional numerical modeling of the system components, (a) steel ring model; (b) wire rope model; and (c) the assembled model.

The contact problem in the 3-D FE model was composed of the tangential behavior with the non-friction condition and the normal behavior with the hard contact condition. The contact conditions must develop when the $16 \mathrm{~mm}$ diameter of wire rope comes in contact with the hole of the steel ring according to extent of increase in area of wire rope. However, local stress concentration occurs when 
the shell element gets in contact with the truss element. As, the truss element intrinsically ignores the geometric conditions in this model; thus, to avoid this phenomenon, the contact area of the shell element through which the truss element passes was modelled as a rigid body in this study (shown as the gray areas on the steel ring in Figure 6). MPC link was applied in the modeling to simulate the behavior of U-clip in brake frame. The properties of 3-D FE model for the components of brake frame are given in Table 1. A SS400 steel ring having a Young's modulus of 210 GPa and a yielding stress of $775 \mathrm{MPa}$ was numerically modelled by using the elasto-plastic property with strain hardening and the failure of elements was evaluated by using displacement criterion. The wire rope (IWRC) for the elasto-plastic material has the Young's modulus of $150 \mathrm{GPa}$, a yielding stress of $1000.2 \mathrm{MPa}$ and an ultimate stress of $1336.7 \mathrm{MPa}$ at 0.0494 of strain. The effective cross-sectional area comprised of $58 \%$ of the actual cross-sectional area.

Table 1. Properties of 3-D FE model.

\begin{tabular}{ccc}
\hline Property & Steel Ring & Wire Rope \\
\hline Young's modulus $(\mathrm{GPa})$ & 210 & 150 \\
Yielding stress $(\mathrm{MPa})$ & 775 & 1000.2 \\
Density $\left(\mathrm{kg} / \mathrm{m}^{3}\right)$ & 7850 & 8730 \\
Thickness $(\mathrm{mm})$ & 4 & - \\
Cross-sectional area $\left(\mathrm{mm}^{2}\right)$ & - & 116.62 \\
\hline
\end{tabular}

The loading condition of the 3-D FE model was set to be the same as that in quasi-static load tests, and was set to apply horizontal displacement at both ends of the wire ropes resulting in the development of tensile forces, whereby, the vertical and transverse displacements were fixed. No specific boundary condition was applied to the steel ring except the contact area with wire rope. When the wire rope was subjected to tensile behavior, the buckling and fracture of the steel ring were set to occur according to the contact condition in the hole.

\subsection{Behavior of Brake Frame and Verification of Three-Dimensional Model}

In order to comprehensively analyze the behavior of the steel ring and to verify the numerical results using experimental results, the displacement in the range between phase 2 and 4 was considered while excluding the effect of the resistance of U-clips from the relationship between the tensile force and the displacement of the brake frame. The comparison of the 3-D numerical modeling results, related to the relationship between tensile force and displacement, with the experimental results and the behavior of steel ring during different phases are shown in Figures 7 and 8 . The numerical analysis results match satisfactorily with the experimental results. The 3-D numerical simulations show that the steel ring could withstand a tensile force of $13.6 \mathrm{kN}$ due to primary elastic buckling at a recorded displacement of $479.2 \mathrm{~mm}$. Further, the displacement in the brake frame continued to increase during the phase of plastic collapse and a tensile force of $6.6 \mathrm{kN}$ was recorded at the displacement value of $687.2 \mathrm{~mm}$ due to local buckling on the left shoulder. Furthermore, a tensile force of $10 \mathrm{kN}$ was recorded at the displacement value of $980 \mathrm{~mm}$ due to local buckling on the right shoulder. A difference was observed between the tensile force values obtained from the experimental and numerical results in the displacement range of $600 \mathrm{~mm}$ and $700 \mathrm{~mm}$. The observed difference was due to the fact that the elastic buckling on both the shoulders of steel ring was observed to be occurring together during the quasi-static load tests, as compared to the numerical simulations, in which it happened alternatively. Due to the secondary elastic buckling, a tensile force of $30 \mathrm{kN}$ was recorded at the displacement value of $1057.7 \mathrm{~mm}$ and the behavior of the steel ring was observed to be similar to phase 3 behavior (deformation shape) shown in Figure 8. A tensile force of $54.3 \mathrm{kN}$ was recorded at the displacement value of $1105.7 \mathrm{~mm}$ due to the tensile failure of the steel ring, which was observed to be matching with the experimental results. 
In this study, the brake frame is devised to absorb the impact load on wire rope through buckling and fracture of steel ring as well as by ensuring the additional plastic deformation. When the 3-D FE model was used, it was observed that the tensile force could vary (difference in the numerical and experimental results) in some sections according to order of steel ring buckling; however, when the analysis range up to the fracture point was considered in the model, the accurate behavior of steel ring, as well as the energy absorption rate, could be estimated.

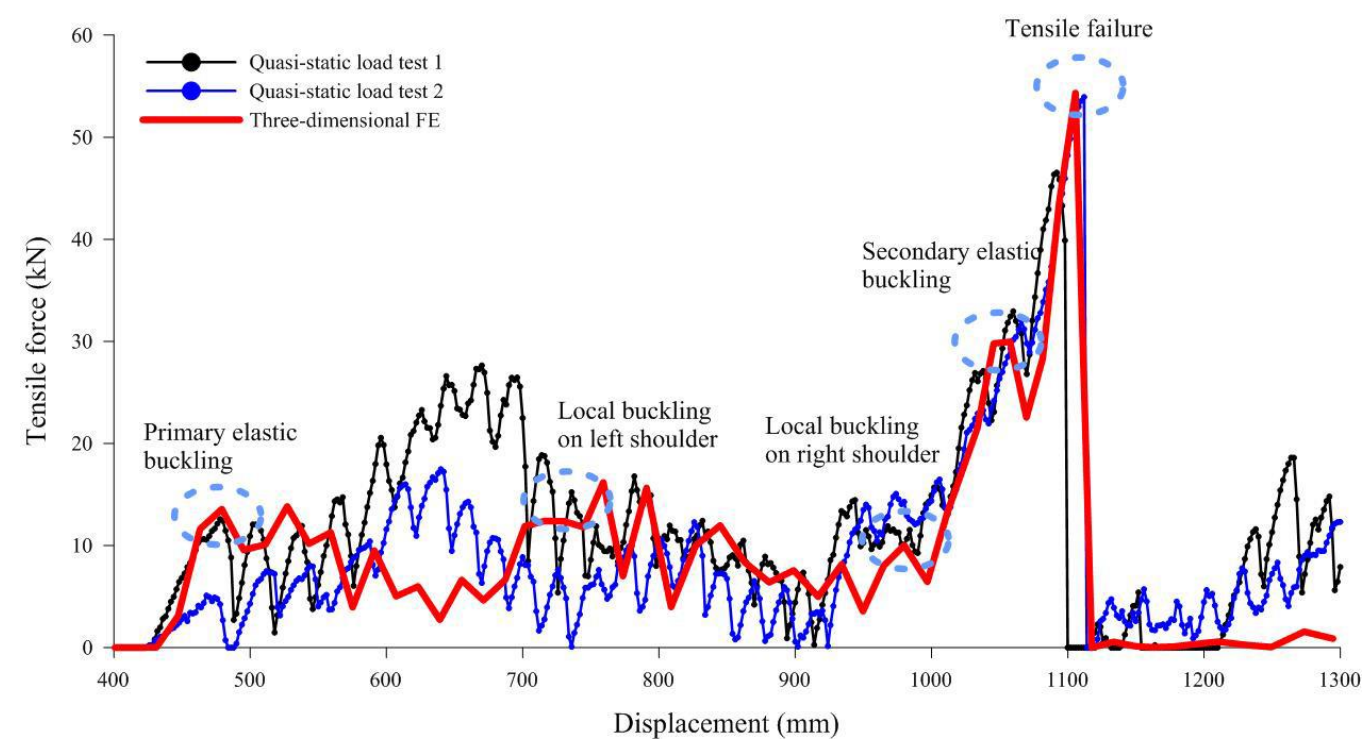

Figure 7. Comparison of three-dimensional model numerical results with experimental results.

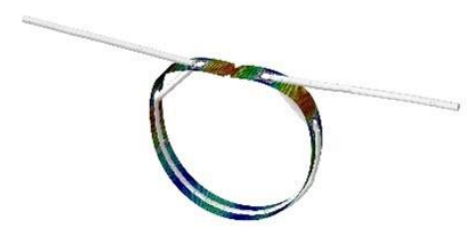

(a)

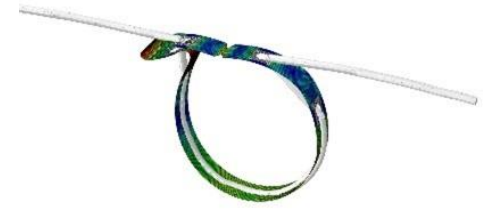

(b)

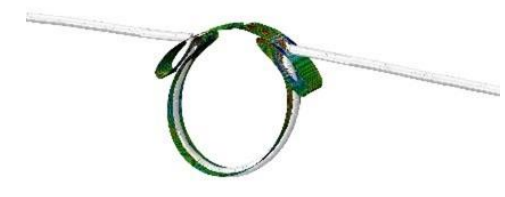

(c)

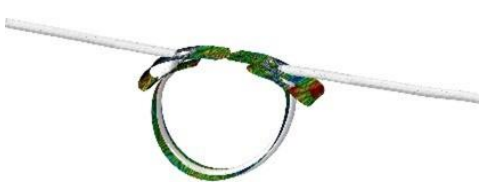

(d)

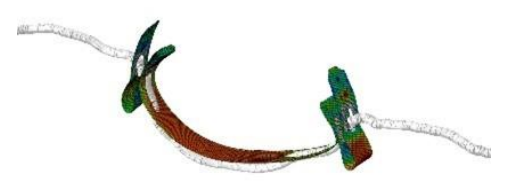

(e)

Figure 8. Three-dimensional behavior of steel rings during different phases, (a) primary elastic buckling; (b) local buckling on left shoulder; (c) local buckling on right shoulder; (d) secondary elastic buckling; and (e) tensile failure.

\subsection{Equivalent Model Based on One-Dimensional Connector for Brake Frame}

The brake frame is an important component of the net protection system and it is necessary to inspect its performance and behavior using full-scale tests or FE analysis. Full-scale testing has various limitations such as the difficulty in conducting repetitive tests and the simulation of various testing conditions. When the 3-D FE analysis, as explained in Section 3.1, is performed for the verification process, the efficiency of analysis also gets decreased. In order to overcome these limitations, an equivalent model based on one-dimensional axial connector is proposed in this study which is capable 
of replacing the 3-D FE model for brake frame. The equivalent model mentioned above is the axial connection type based on nonlinear elasticity and has seven-linear force-displacement relationship, as shown in Table 2. The length of the equivalent model is determined as $0.3 \mathrm{~m}$ based on the equivalent diameter of steel ring. Various points were marked on the force-displacement relationship deduced from the maximum force of different phases. Points 1 and 2 define the gap initially present between the wire rope and steel ring, and represent the incapability of the system to withstand any external force. Points 2-5 represent the system resistance of external force by progressing through the elastic buckling to the tensile fracture stage of the steel ring. Points 5-8 represent the resistance of external force by the wire rope. The results of quasi-static load tests depict the behavior at points 3-4 as well as points 5-6 in a way that either there is a negative slope or there is a decrease in force at these points. However, in order to maintain the stability of the equivalent model, force-displacement phases between these points were replaced with perfectly plastic conditions in the numerical simulation.

Table 2. Force-displacement relationship for equivalent model.

\begin{tabular}{cccc}
\hline Point & Displacement $\mathbf{( m m )}$ & Force $\mathbf{( k N )}$ & Remarks \\
\hline 1 & 0 & 0 & Initial gap (Points 1-2) \\
2 & 250 & 0 & \\
3 & 650 & 45 & Steel ring (Points 2-5) \\
4 & 900 & 45 & \\
5 & 1100 & 70 & Wire rope (Points 5-8) \\
\hline 6 & 1400 & 70 & \\
\hline 7 & 1476 & 160 & 180 \\
\hline
\end{tabular}

Figure 9 presents the results of the quasi-static load tests, the force-displacement relationship of the equivalent model and also the analysis results from the application of equivalent model. Pull displacement was applied at the ends of axial connector to satisfy the equivalent conditions of quasi-static load tests for the simulation of application of equivalent model. The equivalent model which uses a 1-D axial connector, as shown in Figure 9, was observed to be able to accurately simulate the performance of brake frame as well as perfectly replace the complex contact conditions and fractural behavior in the 3-D FE model resulting in a higher efficiency of analysis.

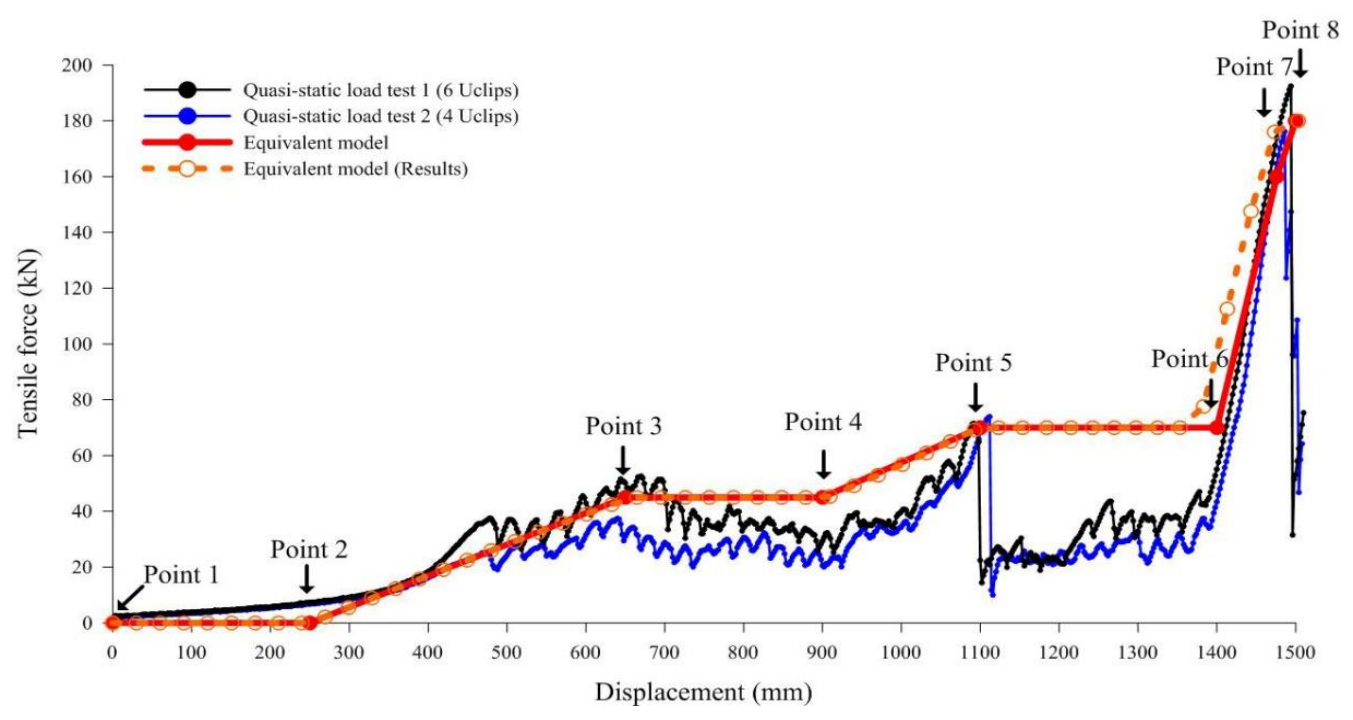

Figure 9. Representation of the equivalent model derived from quasi-static load tests and numerical analysis results using the model. 


\section{Validation by Numerical Simulation and Results}

\subsection{Modeling of the Net Protection System}

The effects of the developed brake frame on the net protection system were numerically validated through ABAQUS Explicit using a specific case in which a spherical impact block of $400 \mathrm{~kg}$ mass and $1 \mathrm{~m}$ diameter was rolled down a slope at an angle of $54.34^{\circ}$, as shown in Figure 10. The slope and the impact block were modelled as rigid bodies. The height and width of slope were modelled as $20 \mathrm{~m}$ and $14.35 \mathrm{~m}$, respectively. The impact energy was assessed to be $30 \mathrm{~kJ}$ based on the potential energy related to the height of $7.65 \mathrm{~m}$. The distance between the base of the slope and the net protection system was set as $0.25 \mathrm{~m}$. Factors like height and velocity of the impact block while rolling are shown in Figure 11 . The coefficient of friction of the slope was 0.60 and the speed of impact block just before the impact was measured as $10 \mathrm{~m} / \mathrm{s}$. The net protection system was assembled using horizontal and vertical wire ropes crossing over each other. A total of seven wire ropes were installed horizontally and twelve wire ropes were installed vertically, both at a distance of $0.5 \mathrm{~m}$ between each other. The wire rope was modelled as a two-noded linear 3-D truss element (T3D2), and the spacing between the individual elements was set as $25 \mathrm{~mm}$. The number of elements constituting the wire rope without and with a brake frame were 3176 and 3260, respectively. The physical properties and the sectional area for the modeling of wire rope were used the same as in Section 3.1. There were no specific boundary conditions applied to the twelve vertical wire ropes and their only role was to prevent the horizontal wire ropes from breaking away due to the impact. The seven horizontal wire ropes were designed to withstand the impact due to the hinged boundary conditions at both ends of horizontal wire ropes. Further, the assembly was then analyzed by attaching brake frames to the seven horizontal wire ropes and a comparison was made between the results obtained by modeling the horizontal wire ropes with and without installing brake frames. In the numerical simulation, the brake frames were applied at the center of the horizontal wire ropes only, as shown in Figure 10b, and the proposed equivalent model explained in Section 3.3 was applied afterwards. In order to facilitate the contact condition of impact block with the equivalent model, the $0.3 \mathrm{~m}$ length of brake frame installed in each of the horizontal wire rope was divided in two parts which were connected to each other through a series of spring connections. The impact force was observed to be following the force-displacement relationship in Table 2, causing half of the displacement because the brake frame was divided in two parts.

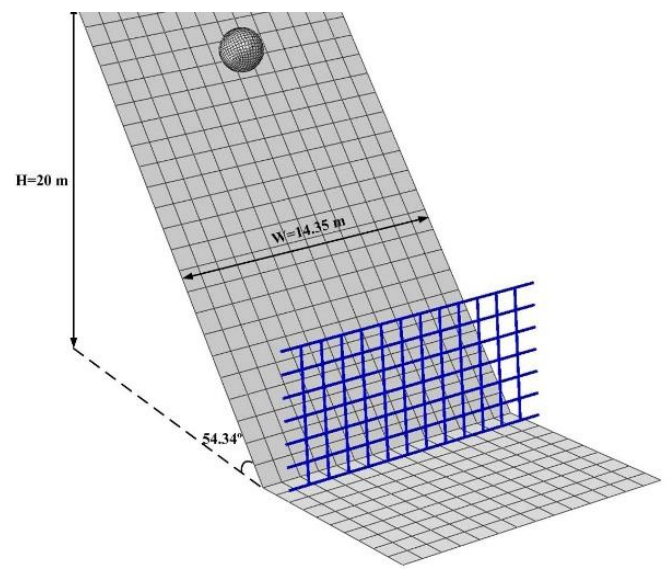

(a)

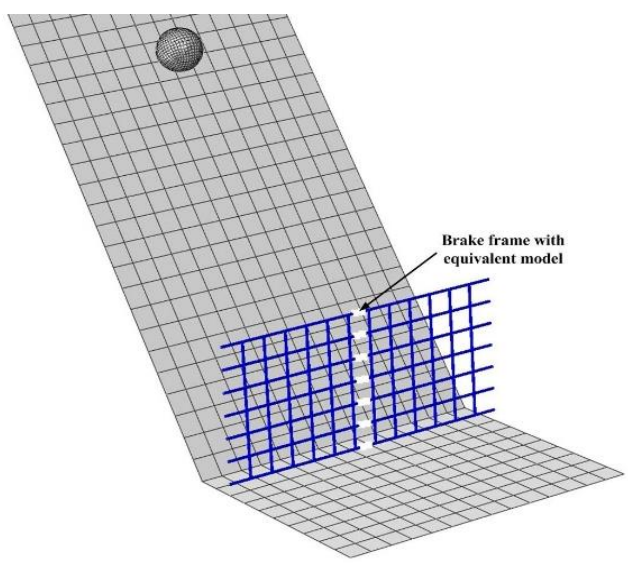

(b)

Figure 10. Numerical models with and without using brake frames, (a) without brake frame; and (b) with brake frame. 


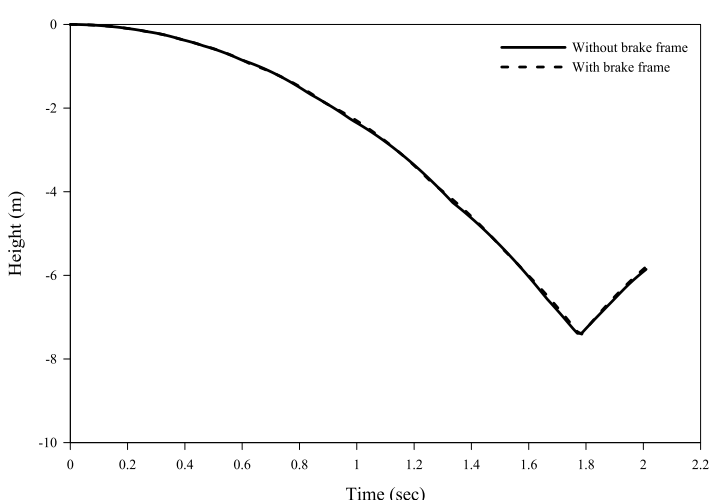

(a)

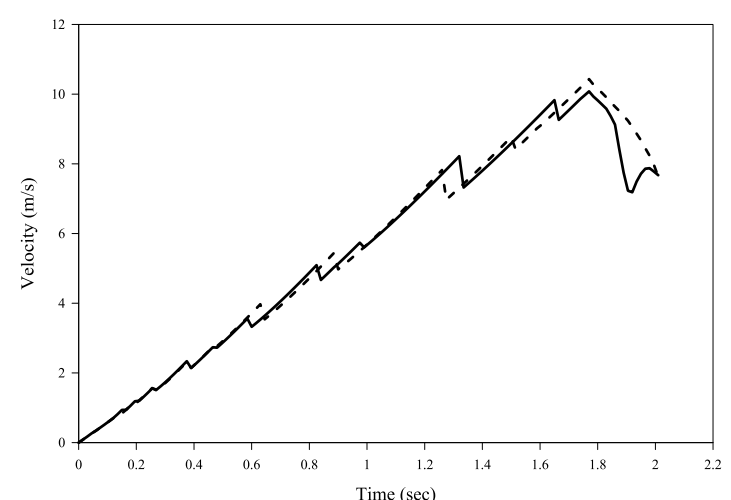

(b)

Figure 11. Height and velocity of impact block while rolling, (a) height of impact block; and (b) velocity of impact block.

\subsection{Effects of Brake Frame on the Net Protection System for Debris Flow Impact}

The horizontal wire ropes were numbered from 1 to 7 starting from the bottom wire rope. The tensile forces obtained at the end of wire rope without brake frames according to time are shown in Figure 12. Without installing brake frames in the net protection system, the maximum tensile forces (reaction force) among all seven wire ropes were obtained to be $117.8 \mathrm{kN}$ and $117.0 \mathrm{kN}$, respectively, in wire ropes 3 and 4 . The brake time (time required to attain maximum tensile force) for wire ropes 3 and 4 was observed as $0.09 \mathrm{~s}$ and $0.12 \mathrm{~s}$, respectively, as shown in Figure 12. The stress contours in the net protection system during this process are shown in Figure 13. When the impact block was rolled down the slope, it made an impact with the wire rope 2 in the first step and a tensile force of $32.2 \mathrm{kN}$ was observed to be developed in wire rope 2 . In the next step, the impact block made impact with wire ropes 3 and 4 developing maximum tensile forces in it among all the seven wire. Afterwards, the impact block was observed to be rebounding towards the slope and making another impact with the net protection system due to which the tensile force developed in all the wire ropes except 3 and 4 were observed to be around $25 \mathrm{kN}$, which lasted for $1.2 \mathrm{~s}$ after the second impact. The impact block was observed to get rebounded to a relatively greater extent because it produced relatively smaller displacement in the net protection system.

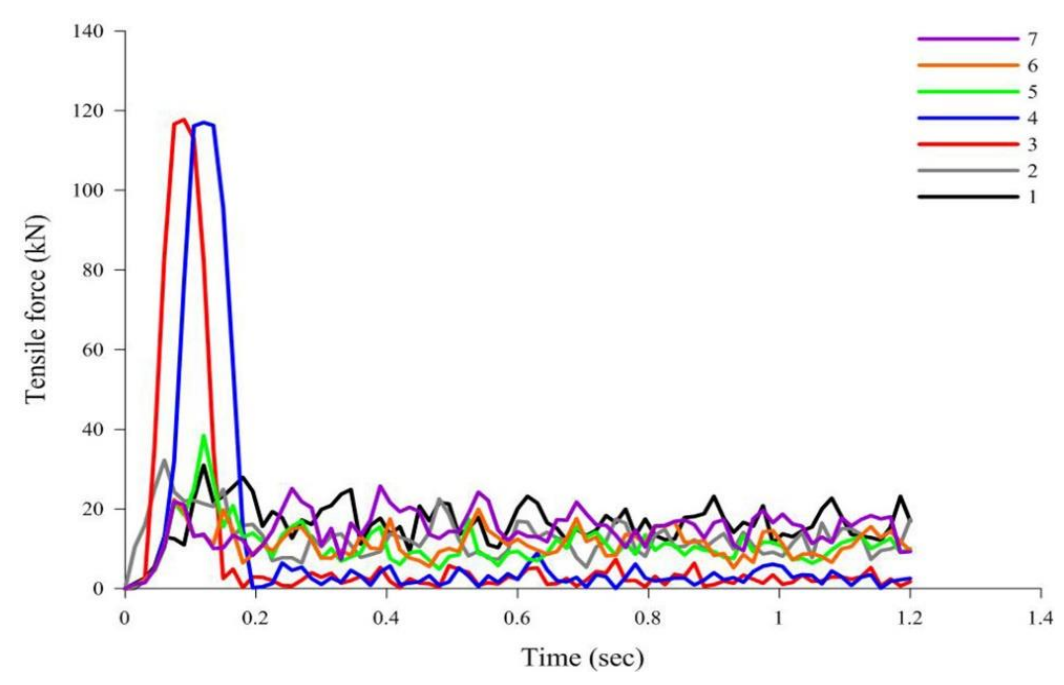

Figure 12. Tensile force acting on seven wire ropes without brake frame. 


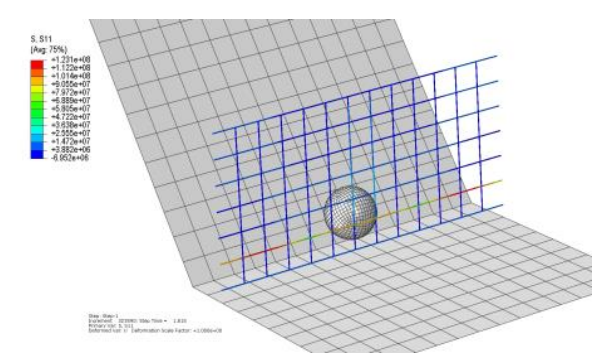

(a)

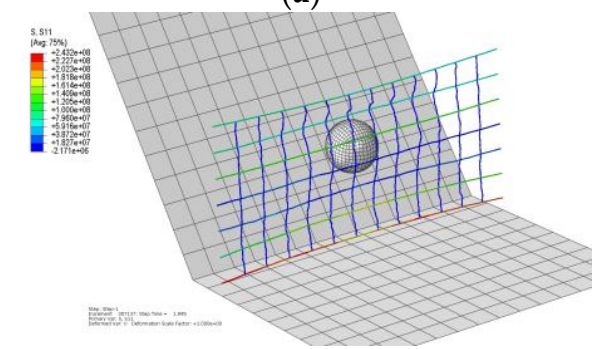

(c)

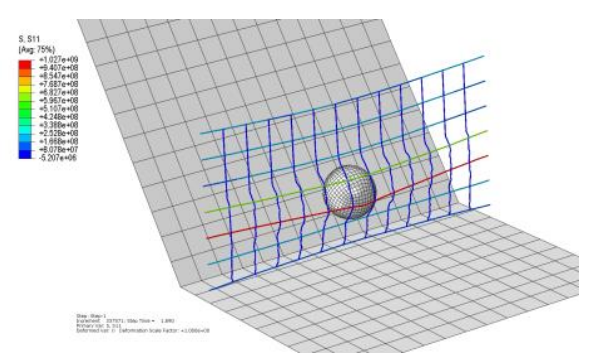

(b)

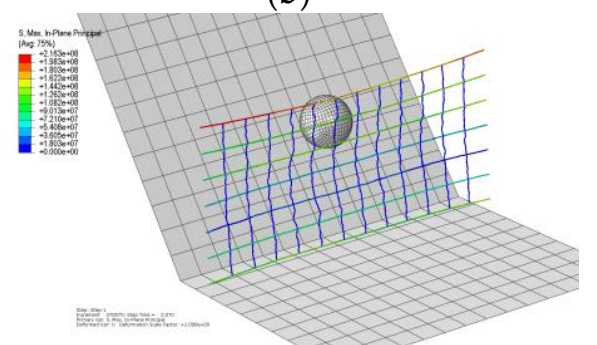

(d)

Figure 13. Stress distribution in the net protection system after impact, without using brake frame, (a) $0.015 \mathrm{~s}$ after impact; (b) $0.09 \mathrm{~s}$ after impact; (c) $0.195 \mathrm{~s}$ after impact; and (d) $0.27 \mathrm{~s}$ after impact.

When the brake frames were installed and the impact block was rolled down the slope, the maximum tensile force developed in wire rope 4 was observed to be $9.9 \mathrm{kN}$ in the brake time of $0.225 \mathrm{~s}$, as shown in Figure 14. Further, the maximum tensile forces were developed in wire ropes 5 and 6 due to the impact and were recorded as $28.4 \mathrm{kN}$ and $29 \mathrm{kN}$ in brake times of $0.27 \mathrm{~s}$ and $0.33 \mathrm{~s}$, respectively. The stress contours in the net protection system during all this process are shown in Figure 15.

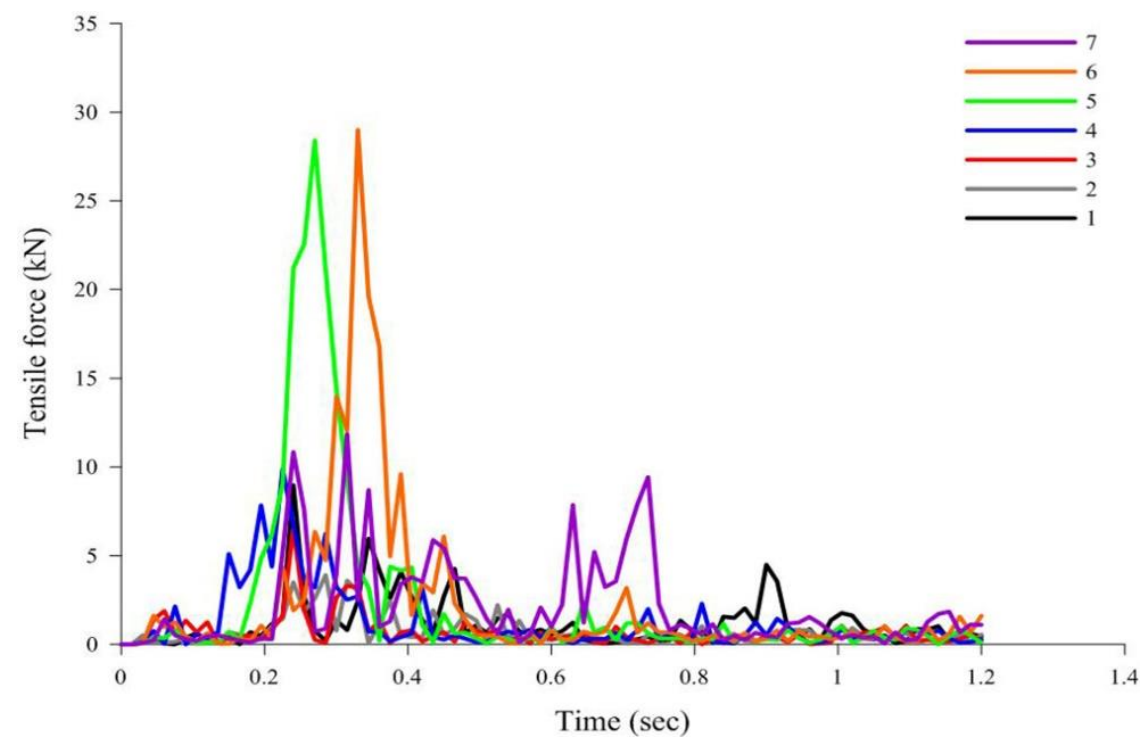

Figure 14. Tensile force acting on seven wire ropes with a brake frame. 


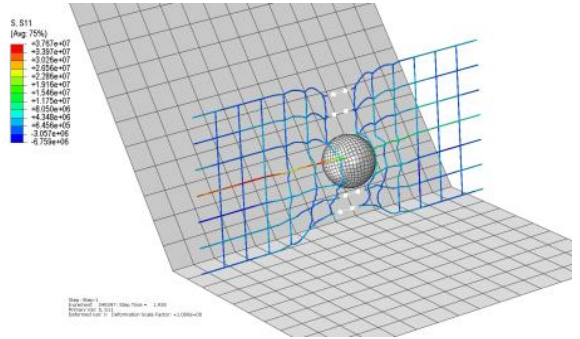

(a)

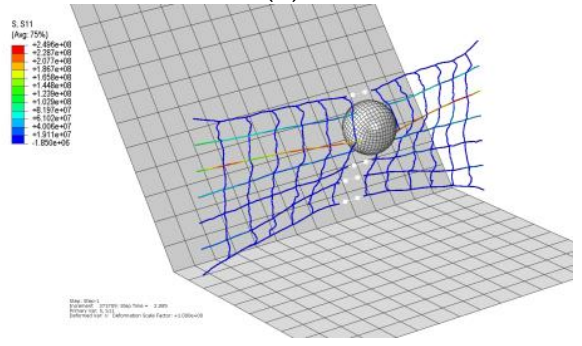

(c)

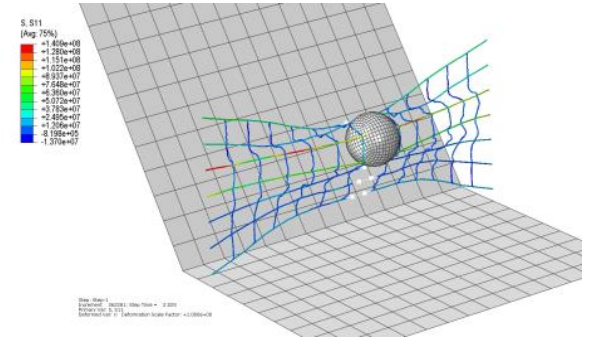

(b)

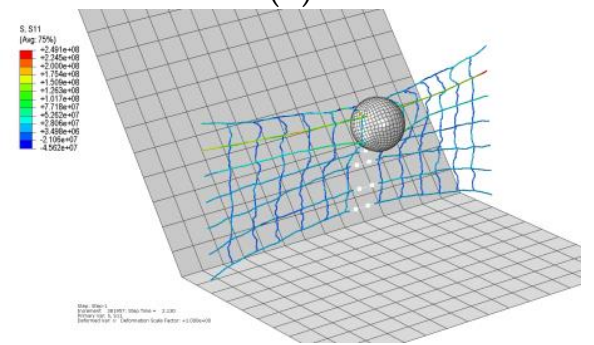

(d)

Figure 15. Stress distribution in the net protection system after impact, with using a brake frame, (a) $0.12 \mathrm{~s}$ after impact; (b) $0.21 \mathrm{~s}$ after impact; (c) $0.27 \mathrm{~s}$ after impact; and (d) $0.315 \mathrm{~s}$ after impact.

\section{Discussion}

The brake frame physically depicts plastic behavior as the displacements cannot be recovered to the initial stages when the external forces are removed after the occurrence of buckling and fracture behavior (phases 1-5, as shown in Figure 4). However, in this study, the proposed equivalent model was axial connection type based on nonlinear elasticity in which the displacements can be recovered to the initial stages after the removal of external forces. Therefore, when the net protection system is analyzed using the proposed equivalent model, the obtained results must be used at the development point of maximum tensile and reaction forces in the wire ropes without considering the results related to restoring forces.

As compared to the cases where the brake frames were used, the tensile forces in each wire rope were observed to be at least 1.4-18 times greater in the system without using a brake frame, except in the case of wire rope 6, as shown in Figure 16. The results show that the maximum tensile forces in the wire ropes obtained in the system with brake frame were reduced by $75 \%$, as compared to those obtained in the system without brake frame. The results also show that the sum of tensile forces in all the wire ropes obtained in the system with the brake frame were reduced by $74 \%$, as compared to those obtained in the system without the brake frame.

When the brake frame is used, the load distribution in the upper wire ropes was observed to increase as compared to the high load distribution in the center portion of the wire ropes when the brake frame was not used due to the reason that the deformation in the wire ropes increases when brake frames were used. In both the cases, the wire rope having maximum tensile force encompassed $28.8-30.6 \%$ of the load distribution. Furthermore, in both cases, two of the wire ropes comprising the most tensile forces acting in them were observed to be encompassing $58-61 \%$ of the load distribution. It is thus deduced from the results that the concentration of the load distribution in some of the wire ropes must be considered for the design of each individual case. 


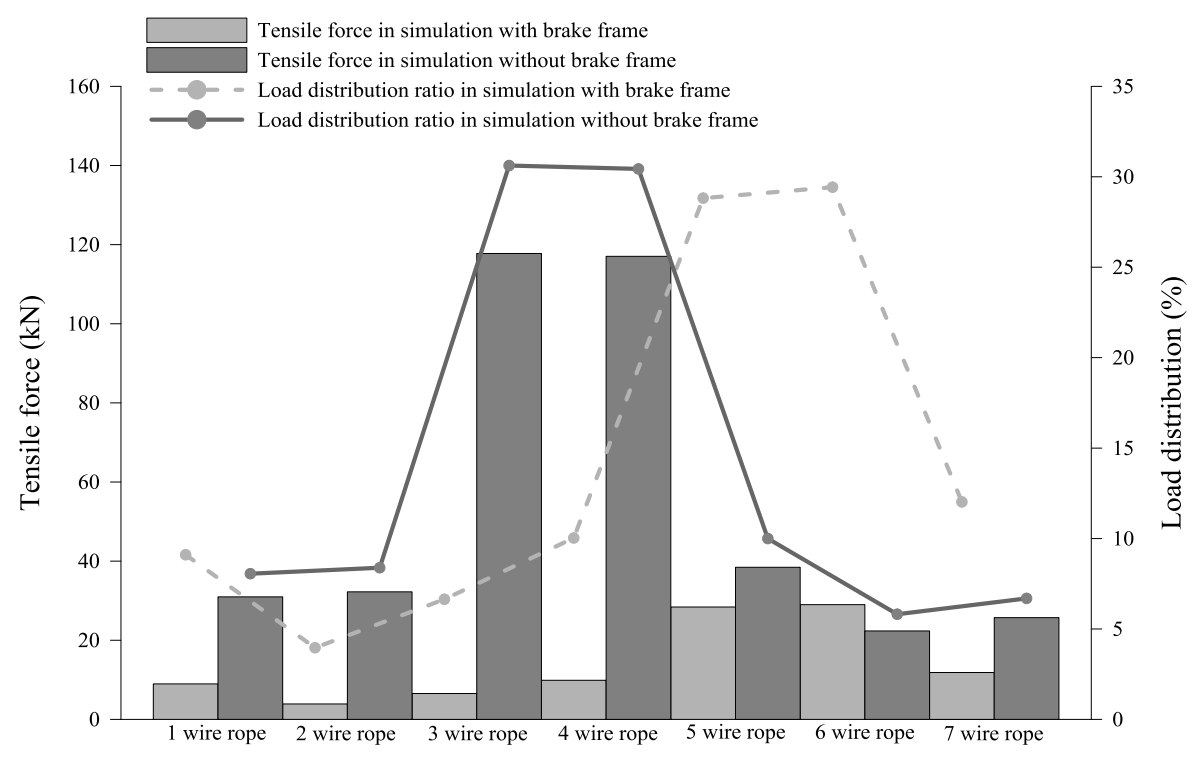

Figure 16. Comparison of the development of tensile forces and load distribution in each wire rope with and without using a brake frame.

\section{Conclusions}

To control the damage caused by debris flow, a net protection system was developed in this study, which is equipped with a new energy absorption device called a brake frame. Deducing the important design parameters was necessary while analyzing the systematic relationship of all elements comprising the net protection system. The quasi-static load tests were performed in order to analyze the performance of brake frames. Using a three-dimensional FE model, the numerical analysis was performed to investigate the behavior of steel rings causing large deformation and the verification of the numerical model was also conducted accordingly. Based on the numerical approach, the effect of the brake frame in reducing the impact load in specific cases was estimated using a proposed equivalent model.

1. The developed brake frames were based on the absorption mechanism of the impact energy through three elements: rings, wire ropes, and U-clips, with six phases of behavioral characteristics. The brake frames could deform up to $1.5 \mathrm{~m}$, thereby absorbing $53 \mathrm{~kJ}$ of impact energy. The field application of the net protection system is possible only when the arrangement as well as the number of brake frames is designed in specific cases according to the impact energy.

2. The steel rings in the brake frame were capable of absorbing the impact energy through the mechanisms of elastic and local buckling, as well as metallic fracture. When the equivalent model based on the one-dimensional axial connector having seven linear force-displacement relationships is utilized, an accurate simulation is performed and an increase in efficiency of the analysis can be expected, as compared to the use of three-dimensional FE model.

3. The anchor capacity was observed to be depending on the performance of the energy absorption devices. The effectiveness of using brake frame in the net protection system was clearly observed because the impact load on the anchor positions (the connection between the wire rope and the ground) was recorded to be about $75 \%$ less than that in the case when the brake frames were not installed. The anchor capacity observed for the specific cases can be used as a reference for the anchor design while using the brake frames in the net protection system.

Extending the observations and conclusions of this study in the future, the relationship between the impact energy and anchor capacity will be developed, and the tensile forces and load distribution in the anchors will be analyzed for various cases of the impact energy based on the full-scale tests. 
Author Contributions: H.Y. supervised the overall process in this study. S.K. designed the net protection system using the brake frame, as well as performed quasi-static load tests. S.H. performed the numerical simulation and proposed the equivalent model. J.K. analyzed the performance and effects of using brake frames, as well as prepared the manuscript by combining the observations of the physical experiments and numerical analysis.

Acknowledgments: This work was supported by the Technology Development Program (S2401940) funded by the Ministry of SMEs and Startups (MSS, Korea).

Conflicts of Interest: The authors declare no conflict of interest.

\section{References}

1. Li, P.; Liu, S.; Lu, Z. Experimental study on the performance of polyurethane-steel sandwich structure under debris flow. Appl. Sci. 2017, 7, 1018. [CrossRef]

2. Osanai, N.; Mizuno, H.; Mizuyama, T. Design standard of control structures against debris flow in Japan. J. Disaster Res. 2010, 5, 307-314. [CrossRef]

3. Gentilini, C.; Gottardi, G.; Govoni, L.; Mentani, A.; Ubertini, F. Design of falling rock protection barriers using numerical models. Eng. Struct. 2013, 50, 96-106. [CrossRef]

4. Gentilini, C.; Govoni, L.; de Miranda, S.; Gottardi, G.; Ubertini, F. Three-dimensional numerical modelling of falling rock protection barriers. Comput. Geotech. 2012, 44, 58-72. [CrossRef]

5. Ferrero, A.; Segalini, A.; Umili, G. Experimental tests for the application of an analytical model for flexible debris flow barrier design. Eng. Geol. 2015, 185, 33-42. [CrossRef]

6. Sasiharan, N.; Muhunthan, B.; Shu, S.; Badger, T. Analysis of global stability, anchor spacing, and support cable loads in wire mesh and cable net slope protection systems. Transp. Res. Rec. J. Transp. Res. Board 2005, 1913, 205-213. [CrossRef]

7. Sasiharan, N.; Muhunthan, B.; Badger, T.; Shu, S.; Carradine, D. Numerical analysis of the performance of wire mesh and cable net rockfall protection systems. Eng. Geol. 2006, 88, 121-132. [CrossRef]

8. Muhunthan, B.; Shu, S.; Sasiharan, N.; Hattamleh, O.A.; Badger, T.C.; Lowell, S.M.; Duffy, J.D. Analysis and Design of Wire Mesh/Cable Net Slope Protection; Washington State Department of Transportation: Olympia, WA, USA, 2005.

9. Cho, S.; Yoo, B.; Kim, J.; Lee, K. Performance evaluation of the anti-earth stone facility using the reduced scale model test. Korean Soc. Hazard Mitig. 2016, 16, 247-260. [CrossRef]

10. Kim, S.; Yoo, H. Estimation of anchor capacity according to impact energy on net protection system for debris flow. Korean Soc. Hazard Mitig. 2018, 18, 155-166. [CrossRef]

11. Gratchev, I.; Kim, D.H.; Chung, M. Study of the interface friction between mesh and rock surface in drapery systems for rock fall hazard control. Eng. Geol. 2015, 199, 12-18. [CrossRef]

12. Shu, S.; Muhunthan, B.; Badger, T.C.; Grandorff, R. Load testing of anchors for wire mesh and cable net rockfall slope protection systems. Eng. Geol. 2005, 79, 162-176. [CrossRef]

13. Simulia, D.S. Abaqus 6.16 Theory Manual; DS Simulia Corp.: Providence, RI, USA, 2015.

(C) 2018 by the authors. Licensee MDPI, Basel, Switzerland. This article is an open access article distributed under the terms and conditions of the Creative Commons Attribution (CC BY) license (http:/ / creativecommons.org/licenses/by/4.0/). 